unspecified, 53 with radiation proctitis, 13 with diversion colitis, 10 with ileo-anal pouchitis and 7 with microscopic colitis. Using the database for reference, the hospital coding system was used to analyse the total number of lower gastrointestinal endoscopy procedures performed between 2001 and 2011 on IBD patients. The total cost for the primary care trust (PCT) was then calculated based on the Department of Health National Tariff System, awarding £561 for a diagnostic colonoscopy and $£ 482$ for a flexible sigmoidoscopy. Results Over the 10 year period, the 2680 patients underwent 1835 flexible sigmoidoscopies (1405 in UC patients, 134 in Crohn's patients and in 296 patients with IBD unspecified) and 1475 colonoscopies (780 in UC patients, 436 in Crohn's patients and in 259 patients with IBD unspecified). In total this cost the PCT $£ 884,470$ for flexible sigmoidoscopy and £827,475 for colonoscopy.

Conclusion Over the course of 10 years the 2680 IBD patients had a total of 3328 lower GI endoscopies (or 332 per year). This cost the local PCT a total of $£ 1,711,945$ (or $£ 171,194$ per year). A greater utilisation of faecal calprotectin for the assessment of disease activity and treatment monitoring in IBD may help reduce the total number and cost of lower GI endoscopy in this cohort of patients.

Disclosure of Interest None Declared.

\section{PTH-157 THE USE OF A FAECAL CALPROTECTIN IN ROUTINE CLINICAL PRACTICE CAN HELP AVOID DILEMA AND SIGNIFICANTLY REDUCE UNNECESSARY COLONOSCOPY}

doi:10.1136/gutjnl-2013-304907.644

1."M W Johnson, 'T Cacciattolo, 'S Shieh, 'K Lithgo, 'T Price. 'Gastroenterology, Luton \& Dunstable University Hospital, Luton, UK

Introduction The new faecal calprotectin (FC) assessment kits are capable of differentiating between organic and functional bowel disease with a $93 \%$ sensitivity and $96 \%$ specificity (Rheenen. BMJ.2010). Where the diagnosis is unclear, FC can be used to spare unnecessary invasive colonoscopy. Functional (Irritable bowel syndrome - IBS) symptoms occur in $60 \%$ of ulcerative colitis (UC) and $40 \%$ of Crohn's disease patients (Keohane. AJG. 2010). This can cause a notoriously difficult management dilemma, which in turn can lead to over treatment of presumed flares in the inflammatory bowel disease (IBD).

Objectives 1) To assess the ability of FC in differentiating between functional and organic disease where the diagnosis was uncertain, and to review the number of potentially unnecessary colonoscopies that could be spared. 2) To assess the management outcome in symptomatic IBD patients when using FC to determine IBS from inflammatory symptoms.

Methods Over a 6month period FC data was collected from both new gastroenterology referral patients and known IBD patients, where a colonoscopy was being considered because of diagnostic uncertainty about whether they were suffering from organic or functional (IBS) symptoms. A retrospective review was them performed to assess the diagnostic and management outcome.

Results In total $100 \mathrm{FC}$ assessments were performed in new referral patients and 44 in known IBD patients where there had been a diagnostic dilemma. In the new patients colonoscopy was spared in $70 \%(70 / 100)$, including 55/63 with normal FC $(<60)$, 6/7 with borderline FC (60-100), and 9/30 with high FC (>100). Some of these patients did however opt for a CT cologram were positive findings were seen in $0 / 6$ of those with normal FC, $1 / 2$ (1 diverticular disease) with borderline FC, and 7 ( 2 normal, 4 diverticular disease, 1 cancer) with high FC. Despite normal FC results 6 new patients went on to have a colonoscopy, 5 of which were normal and $1 \mathrm{dem}$ onstrated a low grade dysplastic tubular adenoma. In the IBD patients colonoscopy was spared in $84 \%$ (37/44), including 13/14 with normal FC, $8 / 9$ with borderline FC and 16/21 with high FC. In the IBD cohort the FC changed management in $10 / 14$ with normal results, $4 / 9$ with borderline results and $16 / 21$ with high results.
Conclusion With the increasing demand being made on colonoscopy throughout UK, a greater utilisation of faecal calprotectin into clinical practise could help safely relieve some of this burden. Faecal calprotectin can be strongly influential in the management of known IBD patients, and provides confidence for clinicians to focus in on treating functional bowel symptoms and tailor down escalating management regimes in those with normal results.

Disclosure of Interest None Declared.

\section{PTH-158 ALCOHOL IN-PATIENT DETOX: WITHDRAWING THE BURDEN OF IN PATIENT MANAGEMENT}

doi:10.1136/gutjnl-2013-304907.645

1."N Barry, 'M Vinayaga-Pavan, 'R Turner, 'V S Wong. 'Gastroenterology Medicine, Whittington Health, London, UK

Introduction In 2009-10, there were 1,057,000 alcohol related admissions to a hospitals in England ${ }^{1}$. Currently patients treatmented for alcohol withdrawal require hospital admission for at least 4-7 days, to complete medical detoxification. Previous studies have shown that even those patients who have severe symptoms of withdrawal, after 2-3 days, can be safely treated in an ambulatory environment ${ }^{2}$

The Aim of this study was to identify the current burden of acute alcohol admissions to the medical unit, demographics of patients and the cost effectiveness of integrating an ambulatory care system. Methods Retrospective data from six months of accident and emergency (A\&E) admissions were reviewed from January 2012June 2012. Patients admitted with acute alcohol intoxication or withdrawal were identified and patients notes analysed. Costings for management were estimated using data sourced from Department of Health Reference Costs 2011-12.

Results Of the 203 patients presenting to A\&E with acute alcohol intoxication or withdrawal, 51 patients were treated for acute alcohol withdrawal (22 female, 29 male). The average age of patients was 60 years. $43 \%$ of patients were managed by acute assessment teams, $31 \%$ by gastroenterology, and $25 \%$ by general medicine. 2 patients required management in ITU. Mean length of stay 10.4 days. Of the patients reviewed the mean drinking years 8.03 with an average of 165 units of alcohol drunk per week.

$50 \%$ of patients had with a documented mental health assessment, of which $74 \%$ had documented mental health conditions other than alcohol dependency. Mortality over this period was $0.1 \%$ and readmission within the six month period was averaged at 2.9 admissions.

Cost of current 7 seven day inpatient admission with 4 hours of junior doctor input was costed at $£ 2183.47$ vs a three day admission with 3 three follow up ambulatory appointments and 2 two hours of junior doctor time costing £1352.57.

Conclusion The burden of alcohol related admissions requires an innovative approach to improve patient care and reduce readmissions. Our study has highlighted the possibility of utilising ambulatory care in selected patients as a method of improving care and reducing the cost of admissions to hospital. It also highlighted the importance of identifying patients as high risk of mental health issues. Previous work has identifed the benefits of outpatient withdrawal in abstenence and psychological well being ${ }^{3}$. Subsequent to this study our hospital has piloted an ambulatory care pathway and prospective analysis will be completed in due course.

Disclosure of Interest None Declared.

\section{REFERENCES}

1. www.ic.nhs.uk/pubs/alcohol12

2. Stockwell et al. Home detoxification from alcohol: its safety and efficacy in comparison with in-patient care. ALcohol and ALcoholism 26. 645-650

3. Fleeman et al. Alcohol home detoxification: A literature review. Alcohol and Alco holism 32. 649-656 